Blair, is not generally accepted. Tests by Gueze and Tan with a torsion plastometer, and by Haefeli, have indicated the existence of yield-point and at higher stresses a pronounced tendency for the development of a state of structural disintegration as in triaxial tests. It is considered by several workers that the apparent yield-point is due to measurement inaccuracies and that the true flow-curve is of the thinning thixotropic form observed by Ostwald. Experimental evidence appears to confirm the conclusion reached by Reiner that volume deformation is generally a combination of elastic, viscous and plastic processes. The rate of the water expulsion occurring with volume decrease in saturated soils is governed by Darcy's law.

The large strain behaviour associated with shear. failure may be considered as of an essentially plastic nature, this being generally assumed in the determination of allowable footing loads which correspond to much smaller strains. Simple viscoelastic models are proposed for small strain volume change and shear deformation caused by stresses $<0.8 \tau_{m}$, based on available experimental evidence. On the assumption of isotropy, these models can be combined for any stress and strain conditions. The predicted behaviour for one-dimensional compression, in which a laterally confined cylindrical sample is subjected to a uniform axial stress, is in reasonable, but not exact, agreement with the observed data. The generally used analysis of one-dimensional compression data is based on the assumption that the structure of the clay particle behaves as an isotropic elastic material for any given increment of load. The inclusion of anisotropic considerations is of excessive mathematical complexity.

The study of the rheology of clay soils is still at a preliminary stage, and much detailed experimental work remains to be done before further progress can be made. The use of apparatus enabling precise measurement of the imposed conditions is essential.

The next meeting of the Club will be held at, the Technological Research Station, Spillers, Ltd., Station Road, Cambridge, on May 7, when Mr. R. G. King (Farol Research Engineers) will speak on "Viscoelasticity and the Weissenberg Rheogoniometer". The instrument will be available for the two days following the meeting for practical demonstrations on materials which members of the Club or visitors are invited to submit. Notice of this should be given in advance to the Secretary of the Club at the above address.
H. G. Muller

\title{
PHENOLIC ALKALOIDS
}

A SYMPOSIUM organized by the Plant Phenolics Group was held at the School of Pharmacy, University of London, on January 9, and was concerned with the alkaloids of the opium poppy and of ipecacuanha.

Under the chairmanship of Prof. W. O. James a paper on the distribution and significance of alkaloids in Papaver somniferum was presented by Prof. J. W. Fairbairn (School of Pharmacy, University of London). The work in his laboratory was chiefly a study of the occurrence of alkaloids in relation to the origin and distribution of laticiferous vessels and an analysis of the latex composition using samples drawn at short intervals from unripe capsules. It was confirmed that alkaloids were restricted to laticiferous vessels and also shown that this anastomizing system always occurred in the phloem, but was absent from stamens, ovules, seeds and seedlings up to the cotyledon stage. Latex from capsules had been assayed for morphine, caoutchouc and total solids, and it was shown that rapid changes in the composition of the latex took place in short periods of time such as $2 \mathrm{hr}$. Similar rapid changes had also been shown to take place in the fruit of Conium maculatum (Fairbairn and Suwal). From anatomical and qualitative chemical results it had been suggested that the alkaloids might be products of photosynthesis, secreted into the laticifers and so removed from metabolic circulation. This 'drain-pipe' theory now appeared unlikely owing to the rapid alkaloidal variation, and further work might raise the status of the latex from that of a slowly growing heap of 'metabolic sewage' to that of a living entity, actively participating in the metabolism of the plant.

Biosynthesis of Opium Alkaloids. An account of the studies which have been carried out in several laboratories was given by Dr. A. R. Battersby (University of Bristol), who was responsible for recent work at Bristol involving the feeding of growing plants with tyrosine, labelled specifically with carbon14. Unambiguous degradation of carefully purified alkaloids, isolated from the plant, had shown that the molecule of papaverine was specifically labelled at positions 1 and 3 and morphine at the corresponding positions 9 and 16 . These results were interpreted as showing that two aromatic units with a two-carbon side-chain $(A r-C-C)$ constitute building blocks in the carbon(16) skeleton of the alkaloids studied. Supporting evidence was available by synthesis of norlaudanosoline specifically labelled with carbon-14, which was incorporated into papaverine and morphine by feeding experiments. Furthermore, results of controlled degradation of the latter radioactive alkaloids showed little doubt that the rings $A$ and $C$ of morphine are joined by a phenolic coupling reaction between two aromatic rings. Rate studies on the incorporation of activity into the two alkaloids from carbon-14 carbon dioxide (Rapoport et $a l$.) and from specifically labelled tyrosine (Bristol workers), together with other evidence, had shown that the alkaloids are formed in the order thebainecodeine-morphine. Concluding his account of recent work, Dr. Battersby was able to show that the present evidence was in keeping with the view that the opium alkaloids are products of the shikimic-prephenic acid pathway, the two $A r-\mathrm{C}-\mathrm{C}$ units produced by this route being used to provide the sixteen carbon atoms required for skeletons of the bases. It was probable that the units were different, yet derivable from tyrosine in the living plant.

Chemical Structure and Pharmacological Activity. In the afternoon session Prof. W. B. Whalley (School of Pharmacy, University of London) introduced Dr. N. J. Harper (Chelsea College of Science and Technology), who discussed the relationships between structure and pharmacological activity of morphine and related substances. He stated that morphine had been modified chemically in many ways in 
attempts to reduce undesirable side effects, namely, liability to produce addiction and depressant effect on the respiratory centre, but such modifications had not yet resulted in a compound which had significantly replaced morphine as a clinical agent. The alternative approach of synthesizing fragments of the morphine molecule had resulted in the production of the morphinans and benzomorphans which represented the greatest achievements in this direction. These studies clearly emphasized the importance of the phenolic, alcoholic and basic groupings. Stereochemical factors played an important part in analgesic activity since, with enantiomorphs, one isomer was always more active than the other and in some synthetic analgesics the most active enantiomorphs were related to $\mathrm{D}-(-)$-alanine. Various studies had led to the hypothesis that in the mediation of analgesic activity a three-dimensional fit at a 'receptor site' was involved and stereochemical implications could be important in the separation of desirable and undesirable pharmacological effects. It was probable that the chief factor limiting the intensity of response and duration of effect of morphine and the synthetic analgesics was biotransformation, and in these transformations the phenolic grouping played an important part. $N$-dealkylation also had important pharmacological implications following from two provocative hypotheses; one relating it to analgesic action, and the other to the development of tolerance.

Chemistry and Biosynthesis of Ipecacuanha Alkaloids. Dr. H. T. Openshaw (Wellcome Research Laboratories, Beckenham, Kent) dealt chiefly with the chemistry of the alkaloids and said that they formed a group of inter-related isoquinoline alkaloids of unique structure, the biogenetic relationship of which to the benzylisoquinoline group was of particular interest. The structure of emetine was established by classical methods of degradation, but in its final stages this investigation received powerful support from the developments in biogenetic theory then current; in turn, the biogenetic theory was strongly upheld by the discovery in ipecacuanha of a postulated intermediate in the biosynthesis. Considerable significance was also attached to the stereochemistry of the alkaloids and their relationship to that of the cinchona and indole series. The absolute configuration of each of the four asymmetric centres in emetine had been established, and attention had been paid to the problem of stereospecific synthesis of the alkaloid. A feature of special interest in the chemistry of emetine was its conversion by mild oxidation into a series of dehydrogenation products, the structure of which had been elucidated.

Pharmacologically, emetine was of considerable importance for its amœbicidal activity, and of special interest in that all the stereoisomers and closely related compounds arising from the synthetic work, only one other substance, 2-dehydroemetine, showed comparable activity.

The symposium therefore proved to be well balanced and showed the value of studies on the location and variation in alkaloids of opium, as a background to results obtained using tracer techniques. Classical chemical investigations, in particular with stereochemical considerations, were of benefit to pharmacology and the work on the ipecacuanha alkaloids formed an interesting comparison with studies on morphine and its related compounds.

S. B. Challen

\section{THE NATIONAL PARKS COMMISSION}

T THE twelfth annual report of the National Parks Commission. for the year ended September 30, 1961, besides providing the customary concise and very readable illustrated account of the activities of the Commission during the year, progress in the national parks and with the long-distance routes, is marked by two notes of anxiety *. First, the Commission is anxious that amending legislation to the National Parks Act of 1949 should not be long delayed. Apart from the practical benefit expected to follow the adoption of the recommendations submitted to the Minister, the Commission believes that reappraisal in Parliament of the working of the Act would give welcome encouragement to those whose duty it is to administer the Parks and would help to produce in the public mind a clearer conception of the whole idea of national parks. The second note of anxiety relates to the continuing proliferation of aerial masts throughout the countryside, usually on remote and unobstructed high ground. These increasing coverage demands appear as yet to be entirely unco-ordinated, and while the Commission recognizes the need, it is far from satisfied that the problem of mast-sharing receives adequate consideration.

* National Parks and Access to the Countryside Act, 1949. Twelfth Report of the National Parks Commission for the year ended September 30, 1961. Pp. vi $+91+8$ plates. (London: H.M.S.O., 1962.) 7s. net.
The magnitude of the development issue is shown by the fact that during the year nearly 450 proposals for development affecting landscape beauty in national parks, in areas of outstanding natural beauty, or elsewhere were referred to the Commission by Government departments, park or other local planning authorities, or by amenity organizations. One notable success during the year recorded in the report is the decision of the North-Western Electricity Board to place the entire Upper Borrowdale scheme underground at its own expense, notwithstanding the offer of contributions from the Friends of the Lake District and the official consent to the placing of certain sections of the line overhead. Regarding areas of outstanding natural beauty, the report comments that review of the work done by the local planning authorities indicated that while the preservation of these areas from unsightly development was receiving full attention, insufficient use was made of the opportunities for positive action for the enhancement of natural beauty which would earn payment of the special 75 per cent rate of Exchequer grant. Some progress is reported with the long-distance routes, notably with the Dorset Coast Path, on the South Downs route, and field work on a North York Moors and a Yorkshire Court Path. One of the two remaining major problems of the Pennine Way has been solved, completing the Path within the Peak District National Park. 